\title{
Propuesta de modelo en dinámica de sistemas para la toma de decisiones en la selección de proyectos verdes
}

\section{A Systems Dynamics Model for Decision-making in the Selection of Green Projects}

\author{
Julián Andrés Castrillón Gómez \\ Ingeniero Electrónico, candidato a Doctor en Ingeniería \\ Grupo de Investigación ABC Dynamics, Universidad Nacional de Colombia sede Manizales, Colombia \\ https://orcid.org/0000-0002-1598-137X \\ jacastrillong@unal.edu.co
}

\author{
Johnny Valencia Calvo \\ Ingeniero Electrónico, Magister en Ingeniería y Doctor en Ingeniería (Ciencias de la Computación y de la Decisión) \\ de la Universidad Nacional de Colombia \\ Facultad de Ingeniería, Universidad de Medellín, Colombia \\ https://orcid.org/0000-0003-1821-2039 \\ jovalencia@udem.edu.co
}

Fecha de recepción: 1 de noviembre del 2018 Fecha de aceptación: 19 de noviembre del 2018

Sugerencia de citación: Castrillón Gómez, J. A., y Valencia Calvo, J. (2018).

\section{RESUMEN}

La época de posconflicto que vive Colombia, luego de la firma de los acuerdos de paz entre el gobierno y los grupos armados, ha causado un creciente interés por las necesidades sociales, ambientales y económicas de las comunidades afectadas por el conflicto armado, conocidas en la actualidad como "sujetos de reparación colectiva". Este interés se refleja en las continuas convocatorias del Gobierno Nacional y las gobernaciones locales, dirigidas a empresas y a la comunidad científica, que buscan contribuir a los retos regionales en materia de medio ambiente, biodiversidad y construcción de paz para estas comunidades. Ante el nuevo panorama, se requiere que los procesos de selección de las propuestas y la toma de decisiones se transformen, para que, además de elegir el mejor proyecto, se involucre a la comunidad de forma activa durante el proceso de convocatoria y se causen en ella beneficios relacionados con el aprendizaje social y la apropiación del conocimiento.

Teniendo en cuenta lo anterior, el presente artículo propone la creación de una metodología que involucre diferentes métodos científicos que permitan la participación de la comunidad y el conocimiento de la dinámica social, ambiental y económica, a fin de que el proceso de toma de decisiones en las convocatorias o procesos de 
selección de proyectos verdes se realice con base en el análisis de la información extraída de la misma comunidad. Como técnicas para el desarrollo de la nueva metodología de evaluación y priorización de proyectos verdes, se propone la integración del modelado en sistemas dinámicos, el modelado participativo y el proceso analítico de redes (ANP) para la toma de decisiones. Con los hallazgos de la presente investigación se espera contribuir desde el campo científico al desarrollo de un modelo sostenible y participativo que pueda ser aplicado a la toma de decisiones, así como facilitar la toma de decisiones en los procesos de selección de proyectos verdes que se realizan en el marco del posconflicto en Colombia, desde un enfoque práctico.

Palabras clave: proyectos verdes, evaluación y priorización, participación de la comunidad, toma de decisiones, posconflicto.

\section{ABSTRACT}

Post-conflict era in Colombia after the enactment of the peace agreements between the government and armed groups has caused a growing interest in the social, environmental and economic needs of the communities affected by armed conflict, today known as "collective reparation subjects". This interest is perceived through the continuous calls by national and local governments to companies and the scientific community, which seek to contribute to the regional challenges in terms of environmental conservation, biodiversity, and peacebuilding within these communities. Considering this new scenario, selection and decision-making processes on this issues need to be transformed, so that, in addition to selecting the best projects, communities are actively involved, thus generating benefits related to social learning and knowledge appropriation.

Therefore, the aim of this research is to propose a methodology that involves different scientific methods for enabling community participation and knowledge construction on social, environmental and economic dynamics, in order to incorporate the analysis of information directly obtained from communities in decision-making processes for the selection of green projects. For the development of the proposed methodology for green projects assessment and prioritization, we suggest the integration of dynamic systems modeling, participatory modeling and the analytical network process (ANP). With our results we expect to contribute from a scientific perspective to the development of a sustainable and participatory model that can be applied in decision-making processes. In addition, from a practical approach, we expect this work facilitates decision-making in the selection processes of green projects taking place in post-conflict areas in Colombia.

Keywords: Green projects, evaluation and ranking, community participation, decision-making, post-conflict.

\section{INTRODUCCIÓN}

Ante la nueva realidad que atraviesa Colombia tras la firma del acuerdo de paz con la guerrilla de las FARC y la búsqueda de acuerdos con otros grupos armados, surge un gran interés por el medio ambiente y su conservación. Dicho interés se presenta en especial en las zonas más afectadas por el conflicto armado las cuales han sido identificadas como sujetos de reparación colectiva en el proceso de posconflicto (Unidad Para las Víctimas, 2016). Este nuevo panorama exige que las convocatorias encargadas de evaluar y priorizar los proyectos o iniciativas verdes se transformen, no solo para seleccionar aquel con las mejores calificaciones, sino para que, a través del proceso de selección y toma de decisiones, se logre involucrar a la comunidad afectada, permitiendo la generación de bienestar y aprendizaje social y contribuyendo a la generación de políticas sociales y ambientales que ayuden a llenar vacíos existentes.

En el presente artículo se propone la creación de una metodología que facilite la toma de decisiones en las convocatorias (procesos de selección) de proyectos verdes, mediante el empleo de métodos científicos que, primero, permitan la participación de la comunidad en el proceso, segundo, generen aprendizaje y bienestar social y, tercero, permitan a los expertos evaluadores un proceso de toma de 
decisiones informado a través del conocimiento de la dinámica social, económica y ambiental de la región.

El artículo inicia con la presentación de algunos antecedentes y razones dentro del contexto colombiano por las que debería considerarse un cambio en la toma de decisiones para la selección de proyectos verdes. La siguiente sección presenta la revisión de literatura relevante y la definición de las bases teóricas sobre las que se fundamenta la propuesta. Posteriormente, se da a conocer el modelo propuesto en dinámica de sistemas y, a manera de ejemplo, se analizan algunos de sus flujos. La parte final del documento desarrolla la discusión de los hallazgos y presenta las conclusiones del estudio.

\section{LA IMPORTANCIA DE LA TOMA DE DECISIONES EN LA SELECCIÓN PROYECTOS VERDES}

El asunto considerado en el presente artículo nace de la revisión efectuada a diferentes procesos de selección que se realizan en Colombia con el fin de evaluar y priorizar proyectos verdes. Como resultado de esta investigación, se encuentran varios factores que han hecho que cada vez sea más frecuente la oposición de la comunidad a estos proyectos, entre los cuales se encuentran, en primer lugar, factores asociados a la escasa participación de la comunidad y el desconocimiento de la dinámica ambiental, social y económica de la región; y en segundo lugar, factores asociados a la unificación del modelo con que se evalúan los proyectos, siendo este igual para todo tipo de proyectos y comunidades, omitiendo la forma como se ve afectada determinada comunidad por el desarrollo de proyectos que pudieren no ser de su completo interés o que no cumplen con sus expectativas.

Los procesos de selección de proyectos anteceden a la toma de decisiones por parte de los entes gubernamentales, por lo que es crucial contar con una metodología clara que responda a las necesidades sociales, económicas y ambientales de todas las partes interesadas, tales como el gobierno, la comunidad y las demás instituciones financiadoras, además de aquellas organizaciones que promueven la protección del medio ambiente.
Por lo anterior, el presente artículo se centra específicamente en la evaluación técnico-científica de los proyectos, mas no en el cumplimiento de aspectos financieros, administrativos y legales, por ejemplo, requeridos para la participación en el proceso de selección.

\section{Consideraciones}

Gracias a los acuerdos de paz logrados en Colombia en los últimos años, el interés por el medio ambiente por parte del gobierno, organizaciones privadas y otras no gubernamentales ha crecido a tal punto que pueden observarse diferentes convocatorias que buscan financiar o patrocinar los mejores proyectos verdes. Algunas de estas convocatorias corresponden a iniciativas de gobiernos departamentales y municipales, mientras que otras se realizan con el apoyo de instituciones extranjeras como el Banco Interamericano de Desarrollo (BID) y la Organización de las Naciones Unidas (ONU), siendo canalizadas a través del Gobierno Nacional por medio de Colciencias, entidad encargada de llevar a cabo los procesos de selección, en la mayoría de los casos.

Dada la extensión de los procesos de selección y el interés propio de esta investigación, se ha delimitado el problema a los siguientes aspectos:

Evaluación de proyectos: el presente artículo se centra en el estudio de los métodos científicos que pueden ser aplicados a los procesos de selección (convocatorias) de proyectos verdes en Colombia, de manera que se complemente la evaluación técnica (científica) y se facilite el proceso de toma de decisiones (priorización).

Social: esta investigación estará enfocada en los proyectos verdes dirigidos especialmente a las comunidades categorizadas como sujeto de reparación colectiva en el posconflicto y que además padecen condiciones de pobreza extrema y problemas de salud pública, entre otros, pero que a su vez son de gran interés para las instituciones privadas, ONG y el Gobierno Nacional debido a su alto potencial ambiental y económico para el desarrollo del país (Defensoría del Pueblo, 2014).

Ambiental: para este proyecto se tendrá en cuenta, específicamente, aquellas convocatorias y proyectos relacionados con el uso del agua y el uso del suelo, 
dos de los problemas actuales que afectan con mayor frecuencia algunas comunidades de Colombia, y que causan mayor preocupación debido a sus efectos sobre la población y la economía de las regiones (Defensoría del Pueblo, 2014; El Tiempo, 2015; UNESCO \& Programa Mundial de Evaluación de los Recursos Hídricos, 2006).

\section{Estrategias (causas y consecuencias)}

\section{Causas}

Para determinar las causas del problema se consultaron los términos de referencia de diferentes convocatorias de proyectos verdes realizadas por instituciones como Colciencias, el Gobierno Nacional (programa Ideas para el cambio), la Gobernación de Boyacá (programa Boyacá BIO, en unión con Colciencias y el Instituto Humboldt), y el Municipio de Medellín (convocatoria iniciativas empresariales sostenibles 2018. Programa emprendimiento sostenible metropolitano).

Para el objeto de esta investigación, se consultaron los términos de referencia relacionados únicamente con los criterios de evaluación técnica (científica) de los proyectos. Adicionalmente, se consultó la sección de convocatorias en la página web de Colciencias, haciendo revisión de aquellas cuyo objetivo fuera la selección de proyectos verdes para superar retos ambientales.

De la revisión efectuada de estos procesos de selección puede concluirse que el objetivo principal de estas convocatorias está enfocado al cumplimiento de los objetivos del milenio (IPCC, 2013; PNUD, 2016a, 2016b, 2016c) y de la Agenda 2030 (García-Arbeláez, Vallejo-López, Higgins, \& Escobar, 2016), dados por la ONU en 2016. Igualmente, estas iniciativas buscan dar cumplimiento a las necesidades planteadas en los planes de desarrollo nacional y local, los cuales, a su vez, deben estar en línea con los objetivos de las instituciones internacionales y los gobiernos extranjeros que brindan apoyo a Colombia.

Si bien es claro que estas convocatorias surgieron a raíz de la identificación de necesidades en comunidades consideradas como sujetos de reparación colectiva en el posconflicto, no se logra aún identificar manifiestamente en los procesos de selección de proyectos verdes el uso de métodos científicos sobre los cuales se pueda, primero, determinar las necesidades de las comunidades con la participación activa de la misma, segundo, brindar información a los expertos y tomadores de decisiones acerca de la dinámica social, ambiental y económica de la región, favoreciendo una toma de decisiones acertada, y, tercero, un método científico que permita la evaluación de criterios cuantitativos y cualitativos donde sea posible reducir sesgos y aumentar el grado de objetividad en las decisiones.

\section{Consecuencias}

Con relación a este aspecto, se resalta que, si bien las actuales convocatorias buscan generar beneficios a las comunidades, tales beneficios se postergan hasta la ejecución del proyecto. La metodología propuesta en esta investigación, por el contrario, sugiere que es posible generar beneficios a la comunidad relacionados con el aprendizaje social y la apropiación del conocimiento desde el inicio mismo del proceso de selección. Por otro lado, es de notar que aunque se reclama la participación de la comunidad esta se ve relegada a la etapa de ejecución, e incluso ocurre posterior a su culminación. En consecuencia, son desaprovechados los valores y los conocimientos de la comunidad en el proceso de evaluación y priorización, factores claves que pueden determinar el éxito del proyecto.

Por otra parte, es pertinente mencionar que falta contar con un método científico claro que apoye la evaluación y la toma de decisiones en los procesos de evaluación y selección de proyectos verdes. Estos métodos, muchos de los cuales han sido ya desarrollados y comprobados, facilitan en gran medida los procesos de evaluación y toma de decisiones (Antunes, Santos, \& Videira, 2006). Así mismo, resultan de gran aplicación en ambientes complejos, como el caso colombiano, donde deben ser consideradas múltiples variables y criterios (Reed, 2008), además de factores sociales, ambientales y económicos que pueden afectar en mayor o menor proporción los resultados del proceso (Antunes et al., 2006).

\section{Razones para un cambio en la toma}

\section{de decisiones}

A partir de lo anterior, se evidencia la necesidad de una nueva metodología que facilite la toma de 
decisiones en los procesos de selección y priorización de proyectos verdes. Esta metodología debe contar con la participación activa de las comunidades que se verán afectadas, lo cual es pieza clave para garantizar el éxito del proyecto que resulte elegido. Al respecto, Voinov y Gaddis (2008) afirman que aquellos proyectos que no involucren valores ni conocimientos de los afectados, posiblemente terminarán en modelos incompletos, y tal vez a mediano o largo plazo en conflictos potenciales, incomprensión e incluso litigios.

El concepto de modelización científica adaptado (Korfmacher, 2001) permite que las diferentes opiniones, así como las experiencias vividas y el conocimiento de las características y otros factores claves de la zona, se conviertan en un valioso aporte para construir modelos participativos que alcancen la comprensión global de una problemática ambiental. Al mismo tiempo, se otorga a la comunidad un rol activo donde sea partícipe de la toma de decisiones, lo cual, en consecuencia, genera el apoyo de la comunidad al proyecto seleccionado, incrementando con ello sus probabilidades de éxito (Voinov \& Gaddis, 2008).

De otro lado, Colombia es un país con muchos recursos en materia ambiental (García-Lozada, 2014). Sin embargo, el conflicto armado que ha sufrido durante décadas ha retrasado el progreso en este sentido. La nueva realidad de los acuerdos de paz con los grupos armados abre las puertas para que a través de los proyectos verdes, pueda llevarse el desarrollo a las regiones más afectadas por el conflicto, con el fin de satisfacer sus necesidades básicas y contribuir con una mejor calidad de vida para sus comunidades. En consecuencia, surge la necesidad de diseñar e implementar una nueva metodología de evaluación y priorización de proyectos verdes, que no solo considere la dinámica social, ambiental y económica de la región, sino que además involucre variables cualitativas como los valores y los conocimientos de la comunidad, propendiendo por la generación de beneficios para los miembros de dicha comunidad a través de la consolidación de procesos de aprendizaje social y de apropiación del conocimiento (Johnson et al., 2012).

\section{REVISIÓN DE LA LITERATURA}

\section{Dinámica de sistemas y sistemas dinámicos}

La dinámica de sistemas permite construir diferentes escenarios que posibilitan a un analista o experto tener una percepción organizada del mundo real y visualizar su dinámica. Igualmente, el uso de la dinámica de sistemas en ambientes complejos permite establecer las relaciones no lineales existentes entre los diferentes atributos considerados (económicos, ambientales y sociales). Lo anterior, facilita la modelización y la extensión a otras técnicas matemáticas como los sistemas dinámicos, admitiendo la correlación y el análisis de todas las variables (Ibarra-Vega \& Redondo, 2015).

Por su parte, los sistemas dinámicos están basados en ecuaciones diferenciales ordinarias. El modelador convierteunahipótesis dinámicaen una representación de acciones y flujos, entendiendo hipótesis dinámica como la conceptualización de las relaciones causales, bucles de retroalimentación, retrasos, reglas de decisión, entre otras. Las variables de estado están representadas en las existencias (acumuladores) y los flujos (tasa) son los procesos que influyen en el cambio y en los niveles de almacenamiento (Espinoza et al., 2017; Sterman, 2000). Posteriormente, se utiliza un motor de simulación para ejecutar el modelo numérico y simular el cambio en los valores de acciones y flujos con respecto al tiempo (Ahmad \& Simonovic, 2004).

\section{Modelado participativo}

Las diferentes variantes de la técnica de modelado participativo, llámese modelado de grupo, modelado con interesados o modelado con stakeholders, se basan en el principio de involucrar en la producción científica el conocimiento empírico. De esta manera, a través del seguimiento de la diversidad de argumentos y opiniones, el modelado participativo introduce otras variables, por ejemplo, sociales o políticas, con el fin de obtener una mejor aproximación al sistema real analizado (Pahl-Wostl, 2002). De acuerdo con esto, el modelado participativo plantea un cambio de paradigma donde el dominio de los expertos se extiende para incluir el dominio de los interesados (Argent \& Grayson, 2003; Duncan, 2017). 


\section{Toma de decisiones multicriterio (MCDM)}

El proceso de análisis multicriterio generalmente comprende las siguientes actividades: i) identificar tanto a los decisores finales como a los actores involucrados en el proceso de análisis de decisiones, así como a todas las partes interesadas; ii) realizar la selección de criterios; iii) definir las alternativas; iv) elegir una o varias tecnologías para el análisis multicriterio; v) ponderar los criterios; vi) evaluar el desempeño de las alternativas frente a los criterios; vii) transformar los valores de rendimiento de los criterios en unidades medibles; viii) aplicar la técnica o técnicas de análisis multicriterio seccionadas; $i x$ ) realizar el análisis de sensibilidad; $y x$ ) tomar la decisión final (Chung \& Lee, 2009; Saaty \& Vargas, 2006).

\section{SOLUCIÓN PROPUESTA}

La solución planteada en este trabajo parte de la siguiente hipótesis: una metodología que construya un modelo participativo sostenible permitirá a los expertos conocer la dinámica social, ambiental y económica de una comunidad y, con base en esto, tomar decisiones informadas para evaluar y priorizar las propuestas de proyectos verdes en el marco de los procesos de selección de iniciativas ambientales, realizados por el Gobierno Nacional y otras entidades, encaminadas a superar los retos sociales, ambientales, de biodiversidad y de construcción de paz que han sido identificados en las comunidades determinadas como sujetos de reparación colectiva del posconflicto en Colombia. Al mismo tiempo, este tipo de metodología generará aprendizaje y apropiación del conocimiento en la comunidad escogida. La solución propuesta a este respecto se detalla en la figura1.

Figura 1. Diagrama de la solución propuesta

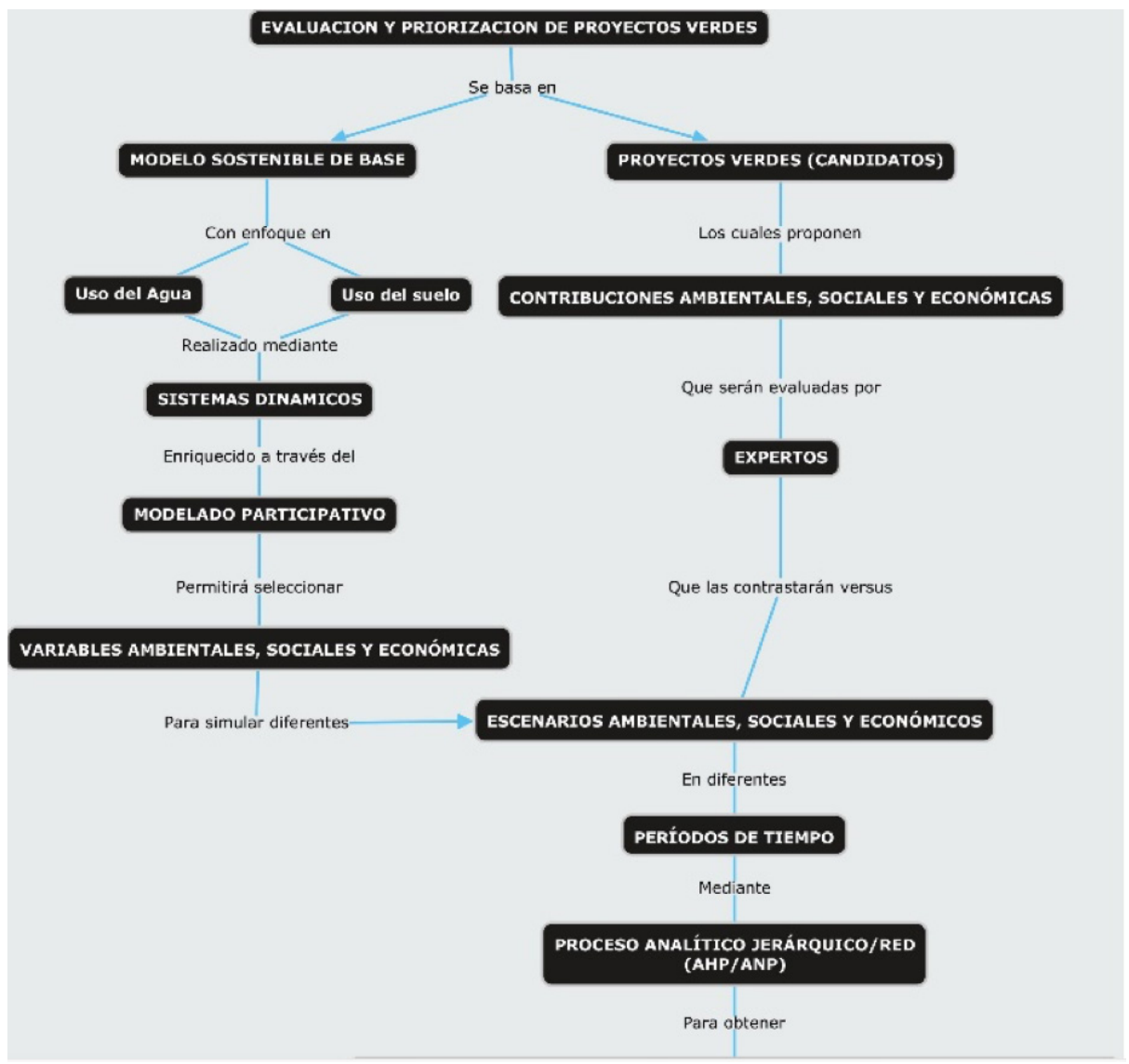


En consecuencia, a partir de la hipótesis planteada será posible construir una nueva metodología para evaluar y priorizar proyectos verdes a través de la elaboración de un modelo sostenible participativo, que facilite la toma de decisiones informadas en los procesos de evaluación y selección de iniciativas verdes en Colombia. Esta nueva metodología debe considerar factores clave como los intereses y las necesidades sociales, ambientales, de biodiversidad y de construcción de paz de las comunidades y, a su vez, podrá convertirse en herramienta para el aprendizaje y la apropiación del conocimiento en la comunidad a quien va dirigida.

\section{Modelo sostenible en dinámica de sistemas}

La primera etapa de la solución total tiene como objetivo específico crear un modelo sostenible a través del cual sea posible conocer la dinámica social, ambiental y económica de la comunidad. Para la construcción de dicho modelo, se tendrán en cuenta las comunidades que hayan sido declaradas como sujeto de reparación colectiva en el posconflicto. De acuerdo con el alcance definido para esta investigación, el modelo se enfocará principalmente en asuntos ambientales relacionados con el uso del agua y del suelo. Finalmente, para la creación del modelo, se propone el uso de la dinámica de sistemas a partir de un modelo de base seleccionado de la literatura (Davies \& Simonovic, 2011).

\section{Modelado Participativo}

La segunda etapa de la solución tiene como objetivo específico hacer del modelo sostenible construido anteriormente un modelo participativo. Para este propósito, se plantea utilizar las técnicas del modelado participativo (Argent \& Grayson, 2003).

\section{Toma de decisiones mediante los métodos de multicriterio (MCDM)}

La tercera etapa de la solución tiene como objetivo específico realizar la evaluación y priorización de los proyectos verdes que hayan sido presentados en un proceso de selección (convocatoria) para comunidades colombianas denominadas como sujetos de reparación. Para este propósito, los expertos tomarán como base para sus decisiones la información resultante del modelo construido en las etapas anteriores.
Adicionalmente, se empleará como método científico el análisis multicriterio para la toma de decisiones, por medio del cual se podrá registrar y tabular los juicios de los expertos. Específicamente, se utilizará el proceso analítico de redes (ANP) desarrollado en 1990 por Saaty (1990), al igual que la escala de evaluación propuesta en esta investigación. El uso de este método permitirá a los expertos evaluar los criterios definidos en el proceso de selección, conociendo de antemano la dinámica social, ambiental y económica de la comunidad y de forma implícita sus valores y saberes, los cuales habían sido considerados anteriormente con el modelado participativo.

\section{Modelo en dinámica de sistemas}

La hipótesis dinámica planteada fue estructurada a partir del diseño de Davies y Simonovic (2011) y se presenta en la figura 2.

En el diseño elaborado se contemplan condiciones sociológicas, ambientales, económicas e institucionales, como se describe a continuación.

Condiciones ambientales: se identifican temas como el uso del agua y del suelo, la calidad y disponibilidad del agua, la huella ecológica y la estacionalidad climática.

Condiciones económicas: en la hipótesis dinámica se considera la producción de la región en relación a sus actividades agrícolas, de minería y de turismo.

Condiciones sociológicas: se considera la dinámica poblacional, la situación actual de la educación, del empleo y de la salud, así como la afectación producida por los incentivos institucionales, los cuales se constituyen como factores clave del modelo.

Condiciones institucionales: la internalización de costos ambientales, las políticas de restricción existentes, la regulación institucional, los impuestos y las restricciones relacionadas con el uso del agua y del suelo.

La hipótesis dinámica planteada hace posible anotar que, por ejemplo, con respecto al uso del agua, esta se ve afectada por las componentes sociales y económicos así: a medida que crece la productividad, la actividad económica de la región es mayor, lo cual ocasiona que tanto la población como el uso del agua 


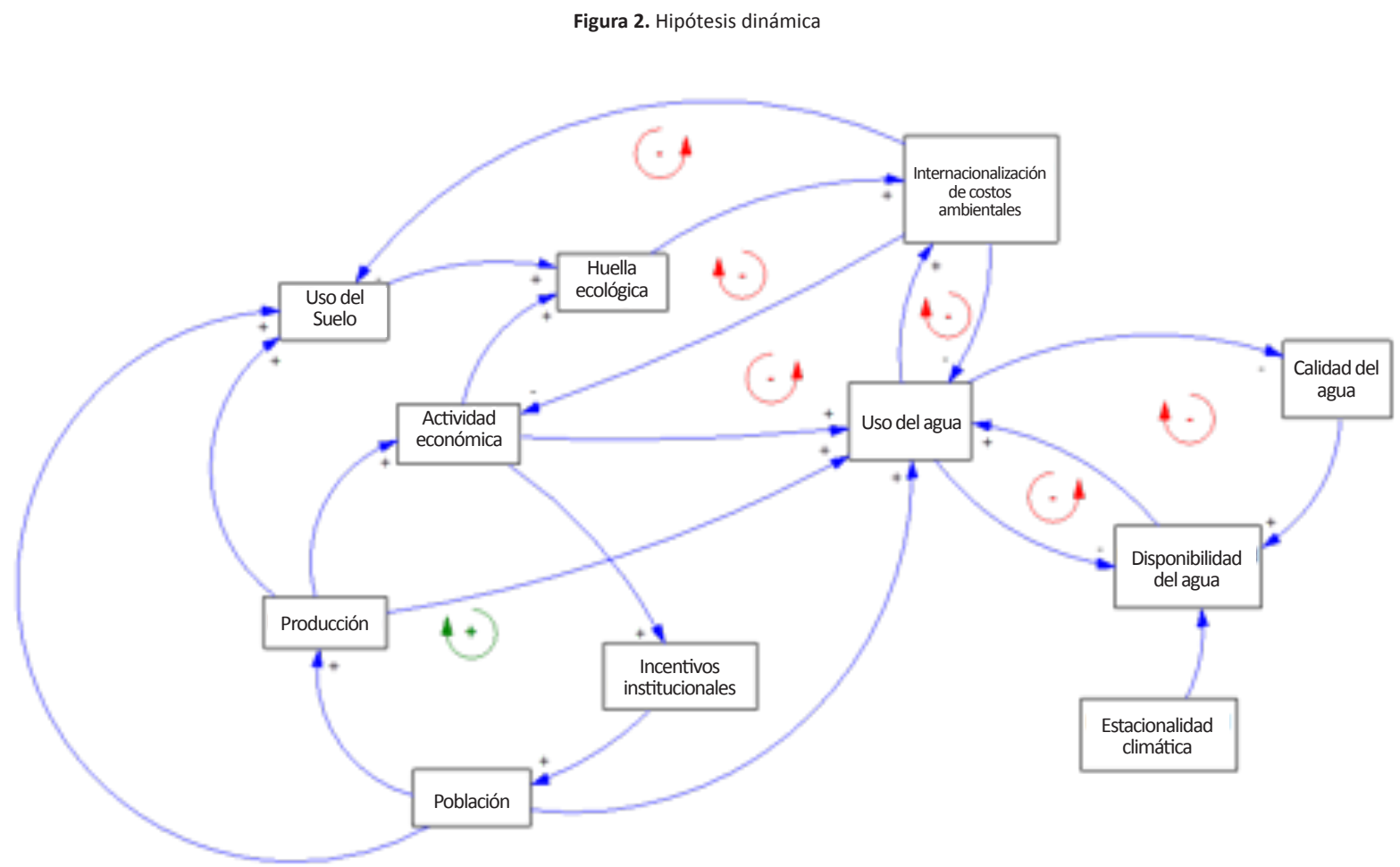

se incremente de forma importante (ciclo de refuerzo). Por otro lado, a medida que incrementa la producción, también aumenta el uso del suelo y, por tanto, la huella ecológica generada; tal situación aumenta los costos ambientales (impuestos) que deben pagarse por la contaminación producida. Este último ciclo actúa como control al uso del agua, con el fin de mantener el equilibrio de manera que no se vea afectada la disponibilidad hídrica de la región.

De otro lado, se puede afirmar que los incentivos institucionales promueven la productividad en la región y contribuyen con el crecimiento poblacional. Sin embargo, los incentivos institucionales pueden verse no solo como estímulos económicos a la población, sino también como aplicación de políticas públicas. Por tal razón, el modelo podría considerarse como un laboratorio de nuevas políticas públicas para analizar sus efectos sobre los demás componentes (económico, social y ambiental).

\section{DISCUSIÓN Y CONCLUSIONES}

Los métodos multicriterio han sido ampliamente utilizados en los procesos de toma de decisiones desde su introducción en la década de 1980. Kulkarni et al. (2004) utilizan este método como alternativa del análisis tradicional de costo-beneficio para priorizar y seleccionar proyectos de mejoramiento de autopistas. Autores como Meade y Presley (2002) muestran que través del método ANP propuesto por Saaty (1990) es posible seleccionar proyectos de investigación y desarrollo, logrando reconciliar los requerimientos de los diferentes interesados.

La propuesta contenida en este artículo está encaminada a lograr la sinergia de los métodos multicriterio y la dinámica de sistemas con las técnicas del modelado participativo sobre las que autores como Tabara y Pahl-Wostl 2007 entre otros, han mostrado ventajas en relación al aprendizaje social y la generación de políticas públicas. Así mismo, esta nueva metodología busca contribuir al desarrollo de un modelo que pueda ser aplicado a la toma de decisiones informadas en la selección de proyectos verdes.

Se espera que con el resultado de la presente investigación sea posible contribuir al diseño de una metodología para la evaluación y priorización de proyectos verdes, en la que sean integrados diferentes métodos científicos que involucren a la comunidad y, además, permitan conocer su dinámica social, ambiental y económica, con el objetivo de que el 
proceso de toma de decisiones sea realizado con base en el análisis de esta información. Así mismo, con esta investigación se busca contribuir al desarrollo de un modelo sostenible y participativo que pueda aplicarse para la toma de decisiones.

En relación al proceso de diseño, desarrollo, validación y aplicación del modelo, esta investigación busca aportar en el campo de la modelización matemática a través del uso de los sistemas dinámicos.

Finalmente, con referencia al proceso de toma de decisiones, se busca aportar en el campo de los métodos multicriterio, llevando a cabo su aplicación en procesos o convocatorias para la selección de proyectos verdes.

En el campo práctico, esta investigación busca proporcionar a las instituciones del gobierno $y$ otras entidades encargadas de realizar procesos de selección de proyectos verdes, una metodología basada en métodos científicos que pueda contribuir a la toma de decisiones informadas en Colombia.

De otro lado, esta investigación busca presentar cómo, desde los mismos procesos de selección de proyectos, es posible generar beneficios a las comunidades por medio del aprendizaje social y la apropiación del conocimiento. Por último, resulta pertinente exponer cómo pueden ser utilizados los modelos participativos para identificar vacíos legales en materia social, ambiental o económica, contribuyendo así a la generación de posibles políticas públicas que puedan subsanarlos.

\section{REFERENCIAS}

Ahmad, S., \& Simonovic, S. P. (2004). Spatial System Dynamics: New Approach for Simulation of Water Resources Systems. Journal of Computing in Civil Engineering, 18(4), 331-340. doi: 10.1061/ (ASCE)0887-3801(2004)18:4(331)

Antunes, P., Santos, R., \& Videira, N. (2006). Participatory decision making for sustainable development - The use of mediated modelling techniques. Land Use Policy, 23(1), 44-52. doi: 10.1016/j.landusepol.2004.08.014

Argent, R. M., \& Grayson, R. B. (2003). A modelling shell for participatory assessment and management of natural resources. Environmental Modelling and Software, 18(6), 541-551. doi: 10.1016/S13648152(03)00029-X

Chung, E.-S., \& Lee, K. S. (2009). Identification of Spatial Ranking of Hydrological Vulnerability Using Multi-Criteria Decision Making Techniques: Case Study of Korea. Water Resources Management, 23(12), 2395-2416. doi: 10.1007/s11269-0089387-9

Davies, E. G. R., \& Simonovic, S. P. (2011). Global water resources modeling with an integrated model of the social-economic-environmental system. Advances in Water Resources, 34(6), 684-700. doi: 10.1016/j.advwatres.2011.02.010

Defensoría del Pueblo. (2014). Acciones para el impulso del derecho humano al agua en colombia: Una mirada crítica al sector de agua potable y saneamiento básico. Bogotá: Defensoría del pueblo. Recuperado de http://desarrollos. defensoria.gov.co/desarrollo1/ABCD/bases/marc/ documentos/textos/Acciones para el impulso del derecho humanos al agua en Colombia.pdf

Duncan, R. (2017). Rescaling Knowledge and Governance and Enrolling the Future in New Zealand: A Co-Production Analysis of Canterbury's Water Management Reforms to Regulate Diffuse Pollution. Society and Natural Resources, 30(4), 436-452. doi: 10.1080/08941920.2016.1265187

El Tiempo. (febrero, 2015). Casi un tercio de la tierra en Colombia está mal utilizada. Recuperado de http://www.eltiempo.com/colombia/otrasciudades/uso-de-la-tierra-en-colombia/16424314

Espinoza, A., Bautista, S., Narváez, P. C., Alfaro, M., \& Camargo, M. (2017). Sustainability assessment to support governmental biodiesel policy in Colombia: A system dynamics model. Journal of Cleaner Production, 141, 1145-1163. doi. 10.1016/j.jclepro.2016.09.168

García-Arbeláez, C., Vallejo-López, G., Higgins, M. L., \& Escobar, E. M. (2016). El acuerdo de parís, Así actuará Colombia frente al cambio climático. Bogotá: Ministerio de Ambiente y Desarrollo Sostenible. 
García-Lozada, H. M. (2014). Alternativas para la gestión del agua en Colombia: La teoría de los sistemas socioecológicos. El Arrendajo Escarlata, 4-12. Recuperado de http://publicaciones. minambiente.gov.co/revista/Documentos_ revista/arrendajo_escarlata_01/arrendajo_01. html

Huang, C., Chu, P., \& Chiang, Y. (2008). A fuzzy AHP application in government-sponsored $R$ \& $D$ project selection. Omega, 36(6), 1038-1052. doi. 10.1016/j.omega.2006.05.003

Ibarra-Vega, D. W., \& Redondo, J. M. (2015). Dinámica De Sistemas, Una Herramienta Para La Educación Ambiental en Ingeniería. Luna Azul, 41, 152-164. doi. 10.1067/mge.2000.105733

Johnson, K. A., Dana, G., Jordan, N. R., Draeger, K. J., Kapuscinski, A., Schmitt Olabisi, L. K., \& Reich, P. B. (2012). Using participatory scenarios to stimulate social learning for collaborative sustainable development. Ecology and Society, 17(2), 9. doi: 10.5751/ES-04780-170209

Korfmacher, K. S. (2001). The politics of participation in watershed modeling. Environmental Management, 27(2), 161-176. doi: 10.1007/s002670010141

Kulkarni, R. B., Miller, D., Ingram, R. M., Wong, C.-W., \& Lorenz, J. (2004). Need-Based Project Prioritization: Alternative to Cost-Benefit Analysis. Journal of Transportation Engineering, 130(April), 150-158. doi: 10.1061/(ASCE)0733-947X(2004)130:2(150)

Intergovernmental Pannel on Climate Change [IPCC]. (2013). Climate Change 2013: The Physical Science Basis. En Stocker, T. F., Qin, D., Plattner, G. K., Tignor, M., Allen, S. K., Boschung, J., ... \& Midgley, P. M. (eds.). Contribution of Working Group I to the Fifth Assessment Report of the Intergovernmental Panel on Climate Change. Cambridge, United Kingdom: Cambridge University Press. doi:10.1017/ CBO9781107415324.

Meade, L. A., \& Presley, A. (2002). R\&D project selection using ANP. IEEE Potentials, 21(2), 22-28.

Pahl-Wostl, C. (2002). Participative and StakeholderBased Policy Design, Evaluation and Modeling Processes. Integrated Assessment, 3(1), 3-14. doi: 10.1076/iaij.3.1.3.7409
Pahl-Wostl, C., \& Hare, M. (2004). Processes of social learning in integrated resources management. Journal of Community and Applied Social Psychology, 14(3), 193-206. doi: 10.1002/casp.774

Programa de Naciones Unidas para el Desarrollo [PNUD]. (2016a). Apoyo del PNUD a la implementación del Objetivo de Desarrollo Sostenible 15 Proteger, restablecer y promover el uso sostenible de los ecosistemas terrestres. New York, USA: ONU

Programa de Naciones Unidas para el Desarrollo [PNUD]. (2016b). Apoyo del PNUD a la implementación del Objetivo de Desarrollo Sostenible 6 Gestión sostenible del agua y el saneamiento. New York, USA: ONU

Programa de Naciones Unidas para el Desarrollo [PNUD]. (2016c). Apoyo del PNUD a la implementación del Objetivo de Desarrollo Sostenible 1 Reducción de la Pobreza. New York, USA: ONU

Reed, M. S. (2008). Stakeholder participation for environmental management: A literature review. Biological Conservation, 141(10), 2417-2431. doi. 10.1016/j.biocon.2008.07.014

Saaty, T. L. (1990). How to make a decision: The analytic hierarchy process. European Journal of Operational Research, 48(1), 9-26. doi: 10.1016/0377-2217(90)90057-I

Saaty, T. L., \& Vargas, L. G. (2006). The Analytic Network Process. Decision Making with the Analytic Network Process, 95(1), 1-27. doi: 10.1007/0-38733987-6_1

Sterman, J. (2000). Business Dynamics: Systems thinking and modeling for a complex world. Irwin/ McGraw-Hill.

Tàbara, J. D., \& Pahl-wostl, C. (2007). Sustainability Learning in Natural Resource Use and Management. Ecology and Society, 12(2), 1-15.

UNESCO \& Programa Mundial de Evaluación de los Recursos Hídricos. (2006). El Agua: una responsabilidad compartida. Segundo informe de las Naciones Unidas sobre el desarrollo de los 
recursos hídricos en el mundo. Recuperado de www.unesco.org/water/wwap/index_es.shtml

Unidad Para las Víctimas. (2016). Reparación Colectiva. Recuperado de http://www.unidadvictimas.gov. co/es/reparación-colectiva/119

Voinov, A., \& Gaddis, E. J. B. (2008). Lessons for successful participatory watershed modeling: A perspective from modeling practitioners. Ecological Modelling, 216(2), 197-207. doi: 10.1016/j.ecolmodel.2008.03.010

Xi, X., \& Poh, K. L. (2014). A Novel Integrated Decision Support Tool for Sustainable Water Resources Management in Singapore: Synergies Between System Dynamics and Analytic Hierarchy Process. Water Resources Management, 29(4), 1329-1350. doi: 10.1007/s11269-014-0876-8 